\title{
Peptidomics analysis revealed that a novel peptide VMP-19 protects against Ang II-induced injury in human umbilical vein endothelial cells
}

\author{
ZHONGQING XU ${ }^{1,2^{*}}$, JINGJING DING $^{2 *}$, LI ZHANG $^{3 *}$, XIANZHEN FENG $^{2}$, \\ JUN ZHOU ${ }^{2}$, XIAOYI SHEN ${ }^{2}$, HONG LU $^{2}$, LINGMEI QIAN ${ }^{2,4}$ and XUN LI ${ }^{1}$ \\ ${ }^{1}$ Department of Cardiology, The First Affiliated Hospital of Soochow University, Suzhou, Jiangsu 215006; \\ ${ }^{2}$ Department of General Practice, Tongren Hospital, Shanghai Jiao Tong University School of Medicine; \\ ${ }^{3}$ Hongqiao International Institute of Medicine, Shanghai Jiao Tong University School of Medicine; \\ ${ }^{4}$ Center for Community Health Care, China Hospital Development Institute, \\ Shanghai Jiao Tong University, Shanghai 200336, P.R. China
}

Received September 26, 2020; Accepted February 2, 2021

DOI: $10.3892 / \mathrm{mmr} .2021 .11937$

\begin{abstract}
Vascular endothelial dysfunction is a vital pathological change in hypertension, which is mainly caused by apoptosis and oxidative stress injury of vascular endothelial cells. Peptidomics is a method for the direct analysis of small bioactive peptides in various biological samples using liquid chromatography-mass spectrometry (MS)/MS. Given the advantages of the low molecular weight, optimum targeting and easy access to cells, peptides have attracted extensive attention in the field of drug research. However, to the best of our knowledge, little is currently known regarding the role of peptides in vascular endothelial injury. In order to investigate the peptides involved in vascular endothelial protection, MS was used to analyze the peptide profiles in the supernatant of human umbilical vein endothelial cells (HUVECs) stimulated by Ang II. The results revealed that 211 peptides were identified, of which six were upregulated and 13 were downregulated when compared with the control group. Subsequently, the present study analyzed the physical and chemical properties and biological functions of identified
\end{abstract}

Correspondence to: Dr Xun Li, Department of Cardiology, The First Affiliated Hospital of Soochow University, 188 Shizi Street, Suzhou, Jiangsu 215006, P.R. China

E-mail:xunli58@126.com

Dr Lingmei Qian, Department of General Practice, Tongren Hospital, Shanghai Jiao Tong University School of Medicine, 1111 Xianxia Road, Shanghai 200336, P.R. China

E-mail:1mqian@shsmu.edu.cn

*Contributed equally

Key words: human umbilical vein endothelial cells, peptidomics, vascular endothelial injury, peptide VMP-19, apoptosis, oxidative stress peptides by bioinformatics, and successfully screened a peptide (LLQDSVDFSLADAINTEFK) named VMP-19 that could alleviate the apoptosis and oxidative stress injury of HUVECs induced by Ang II. In conclusion, to the best of our knowledge, the present study was the first to use peptidomics to analyze the peptide profiles of supernatant secreted by HUVECs, and revealed that the novel peptide VMP-19 could protect HUVECs from apoptosis and oxidative stress injury. The results of the present study could provide novel insights into treatment strategies for hypertension.

\section{Introduction}

An increasing number of studies have shown that vascular endothelial dysfunction is an important pathological change in hypertension, and is also the initial factor underlying vascular injury in hypertension (1). Vascular endothelial cell (VEC) damage is the main cause of hypertension, and its degree of damage is closely associated with the severity of hypertension (2). In turn, hypertension can also cause VEC function damage. When hypertension occurs, VECs are stimulated by the blood flow shear stress and blood flow pulsation, which induces cell damage, resulting in abnormal synthesis and secretion of bioactive substances, and finally, abnormal VEC function (3). Following injury of VECs, numerous active substances are released, such as thromboxane A2 and ADP, which can affect the coordination of endothelin-1 (ET-1)/nitric oxide, increase the synthesis of ET-1, destroy the balance of homeostasis, cause the disorder of vascular tension regulation, and eventually lead to raised blood pressure $(4,5)$. Previous studies have demonstrated that VECs can synthesize and release prostacyclin (PGI2), a vasodilator, which can inhibit platelet aggregation and regulate vasodilation (6). Moreover, PGI2 can inhibit the apoptosis of VECs through peroxisome proliferator-activated receptor (7). Therefore, identifying the bioactive substances secreted by VECs may provide novel ideas for the treatment of hypertension. 
Peptides are a type of small molecule active substance composed of $<50$ amino acids, and are widely involved in various biological functions, such as vasodilatation (8), oxidative stress (9), cell differentiation (10) and apoptosis (11). Due to the advantages of small molecular weight (MW), good targeting and easy-to-enter cells, peptides have attracted increased attention in the field of drug research $(12,13)$. A growing number of peptides are being demonstrated to play a vital role in the clinical diagnosis and treatment of various cardiovascular diseases. For example, atrial natriuretic peptide and brain natriuretic peptide (BNP) have been widely used as biomarkers for screening in patients with heart failure (14), and lyophilized recombinant human BNP has been used in the clinical treatment of heart failure (15). Bradykinin, composed of nine amino acids, not only has vasodilation and hypotensive effects (16), but has also been reported to have protective effects against myocardial ischemia-reperfusion injury (17). An increasing amount of evidence has demonstrated that Apelin, a vasoactive peptide of endogenous ligand of the apelin receptor, has regulatory effects in cardiovascular physiology and pathophysiology, which makes it a potential target for cardiovascular drug discovery and development (18). Therefore, it has been speculated whether VECs may protect against cell injury by secreting certain peptides under stress.

With the rapid development of mass spectrometry (MS), the concept of peptidomics has been widely investigated by researchers in previous years (19). Peptidomics is a novel research method, which can directly analyze small bioactive peptides in various biological samples by liquid chromatography tandem MS (LC-MS/MS) $(20,21)$. In the present study, LC-MS/MS technology was used to investigate the peptides involved in VEC protection stimulated by Ang II. A total of 211 peptides were identified from the cell supernatant, of which, six were upregulated and 13 were downregulated when compared with the control group. Subsequently, the present study analyzed the identified peptides via bioinformatics, and successfully screened a novel peptide, VMP-19, that can alleviate the apoptosis and oxidative stress injury of VECs induced by Ang II.

\section{Materials and methods}

Cell culture and experimental design. Human umbilical vein endothelial cells (HUVECs) were acquired from the Type Culture Collection Committee of Chinese Academy of Science (cat. no. 3142C0001000001250). HUVECs were cultured in Endothelial Cell Medium (ScienCell Research Laboratories, Inc.) supplemented with $10 \%$ fetal bovine serum (Gibco; Thermo Fisher Scientific, Inc.), $1 \%$ penicillin-streptomycin (Wisent, Inc.) at $37^{\circ} \mathrm{C}$ in an incubator with $95 \%$ air and $5 \%$ $\mathrm{CO}_{2}$. When the cell confluency reached $70-80 \%$, the cells were starved in serum-free medium for $6 \mathrm{~h}$, and then treated with Ang II $(1 \mu \mathrm{M})$ for $24 \mathrm{~h}$ at $37^{\circ} \mathrm{C}$. The peptides $(10,20$, 50 or $100 \mu \mathrm{M}$ ) were administered $2 \mathrm{~h}$ before Ang II treatment under the same conditions.

The cell damage model of HUVECs was induced by Ang II (Sigma Aldrich; Merck KGaA). For the initial peptidomic experiments, cells were allocated to two groups: Control group and Ang II $(1 \mu \mathrm{M})$ treatment group. For the subsequent cell experiments, cells were designated to the following groups: i) Scrambled peptide group (Scr); ii) VMP-19 peptide group
(VMP-19); iii) Ang II (1 $\mu \mathrm{M})$ and scrambled peptide cotreatment group (Ang II+Scr); and iv) Ang II (1 $\mu \mathrm{M})$ and VMP-19 peptide cotreatment group (Ang II+VMP-19).

Peptide extraction. The present study collected the cell supernatant of three control groups and three Ang II treatment groups for the peptidomics analysis. The supernatant samples were extracted and ultrafiltrated to $20 \mu \mathrm{l}$ with a $10 \mathrm{kDa}$ ultrafiltration tube at $10,000 \mathrm{x}$. The aforementioned steps were repeated in order to collect the filtered liquid and discard the protein and other macromolecules with a MW $>10 \mathrm{kDa}$ in the ultrafiltration tube. The desalting column was activated with $200 \mu 1$ $0.1 \%$ trifluoroacetic acid (TFA) and $80 \%$ acetonitrile, balanced with 400-600 $\mu 1$ 0.1\% TFA and 1\% acetonitrile solution. The sample was dissolved with $200 \mu 10.1 \%$ TFA aqueous solution and added into the desalting column to make the sample flow through the desalting column slowly. The peptides were captured by the desalting column, and other non-hydrophobic small molecules, such as salt, were discarded. Then, $200 \mu 10.1 \%$ TFA and $0.5 \%$ acetonitrile solutions were added to the desalting column to remove the residual salts. Next, $300 \mu 10.1 \%$ TFA and $80 \%$ acetonitrile solution were added to slowly flow through the desalting column to elute the peptides. The elution solution was collected with a new EP tube, and then freeze-dried.

\section{LC-MS/MS and peptide identification. The nano-LC-MS/MS} on a Q Exactive Plus MS (Thermo Fisher Scientific, Inc.) coupled with a LC1000 was used to identify peptides. Solvent A buffer [Milli-Q (EMD Millipore) water with $2 \%$ acetonitrile and $0.1 \%$ formic acid] and solvent B buffer (90\% acetonitrile and $0.1 \%$ formic acid) were utilized for the chromatographic separation. The samples were resuspended with $20 \mu \mathrm{l}$ solvent A, separated via nano-LC and analyzed using online electrospray tandem MS. A total of $3 \mu \mathrm{l}$ peptide sample was loaded onto the analytical column $(75 \times 250 \mu \mathrm{m}$; Acclaim PepMap C18; Thermo Fisher Scientific, Inc.). The peptides were eluted with $5 \%$ solvent B for $5 \mathrm{~min}, 5-40 \%$ solvent B for $65 \mathrm{~min}, 40-80 \%$ solvent B for $1 \mathrm{~min}, 80 \%$ solvent B for $4 \mathrm{~min}$ and 5\% solvent $\mathrm{B}>20 \mathrm{~min}$ at $300 \mathrm{nl} / \mathrm{min}$. The MS spectra were acquired in the mass range of $350-2,000 \mathrm{~m} / \mathrm{z}$ with a mass resolution of $120 \mathrm{~K}$. The AGC target was set to $1 \times 10^{6}$, and the maximum injection time was $45 \mathrm{msec}$. In total, ten sequential high energy collisional dissociation MS/MS scans with a resolution of $15 \mathrm{~K}$ were acquired in orbitrap. The intensity threshold was 50,000, and the maximum injection time was $35 \mathrm{msec}$. The AGC target was set to $1.0 \times 10^{5}$, and the isolation window was $1.6 \mathrm{~m} / \mathrm{z}$. Xcalibur $^{\mathrm{TM}}$ software (3.1.66.10; Thermo Fisher Scientific, Inc.) was used to perform $10 \mathrm{sec}$ dynamic exclusion, automatic peak identification and tandem MS analysis of the first 20 precursor protein ions at $30 \%$ normalized collision energy.

The intensity of peptides was identified through label-free quantification, and all MS/MS data were analyzed using MaxQuant software (version 1.6.6.0; Max-Planck-Institute of Biochemistry). The Uniprot-SwissProt_homo sapiens database (https://www.uniprot.org/taxonomy/9606; UniProt release 2019-08-09) was used to search and analyze the data. The mass tolerance of the parent ion in MaxQuant was 10.0 PPM and the fragment ion was $0.050 \mathrm{Da} . \mathrm{P}<0.05$ (Student's t-test) and fold-change $>2$ were used as the selection criteria for differentially expressed peptides. 
Bioinformatics analysis. The heat map was drawn using MetaboAnalyst 5.0 software (http://www.metaboanalyst. $\mathrm{ca} /$ ). The MW and isoelectric point (PI) information of the peptides were analyzed online (https://web.expasy.org/protparam/). The precursor proteins of identified peptides were analyzed using the UniProt database. The potential functions of the precursor proteins from the identified peptides were investigated according to the 'Molecular Function', 'Cellular Component' and 'Biological Process' categories in the Gene Ontology (GO) $(22,23)$ and Kyoto Encyclopedia of Genes and Genomes (KEGG) (24) pathways using the Functional Annotation Tool, Database for Annotation, Visualization and Integrated Discovery (DAVID) Bioinformatics Resource 6.8 (https://david.ncifcrf.gov/). Using the Search Tool for the Retrieval of Interacting Genes/Proteins (STRING) database (https://string-db.org/; version, 11.0; medium confidence 0.400 ), the protein-protein interaction networks were generated and analyzed.

Peptide synthesis and administration. The VMP-19 peptide sequence was LLQDSVDFSLADAINTEFK, and its Scr was NLQSVDLLIADTKFELFSD. The present study selected the top six peptides from 19 differential peptides according to the P-value, and these peptides were synthesized by Shanghai Science Peptide Biological Technology Co., Ltd. The purity of all synthesized peptides was $>95 \%$. All peptides were administered $2 \mathrm{~h}$ before Ang II treatment.

Cell viability analysis. Cell viability was detected using a Cell Counting Kit-8 (CCK-8) assay according to the manufacturer's protocol. The HUVECs were seeded in 96-well plates at density of $5 \times 10^{3}$ cells/well, and upon reaching $70-80 \%$ confluency, the cells were starved in serum-free medium for $6 \mathrm{~h}$, and then treated with Ang $\mathrm{II}(1 \mu \mathrm{M})$ for $24 \mathrm{~h}$ at $37^{\circ} \mathrm{C}$. The peptides $(10,20$, 50 or $100 \mu \mathrm{M}$ ) were administered $2 \mathrm{~h}$ before Ang II treatment under the same conditions. After $24 \mathrm{~h}$ of culture, $10 \mu \mathrm{l} \mathrm{CCK}-8$ reagent (cat. no. C0038; Beyotime Institute of Biotechnology) was added to each well and cultured away from light in $37^{\circ} \mathrm{C}$ incubators for $2 \mathrm{~h}$. The intensity of the light absorption at $450 \mathrm{~nm}$ wavelength was measured using a microplate reader.

Lactate dehydrogenase ( $\mathrm{LDH}$ ) detection. The LDH Release Assay kit (cat. no. C0017; Beyotime Institute of Biotechnology) was utilized to detect the level of $\mathrm{LDH}$ release. The reaction working solution was prepared according to the manufacturer's instructions. The cell supernatant (120 $\mu \mathrm{l} /$ well) was collected, mixed with the reaction working solution $(60 \mu \mathrm{l} /$ well) and added to the 96-well plate. The 96-well plate was incubated in the dark at room temperature for $30 \mathrm{~min}$. Finally, the absorbance was detected at $490 \mathrm{~nm}$ wavelength using a microplate reader.

Reactive oxygen species (ROS), superoxide dismutase ( $S O D$ ) and malondialdehyde (MDA) detection. A ROS assay kit (cat. no. S0033M; Beyotime Institute of Biotechnology) was utilized to determine the levels of intracellular ROS according to the manufacturer's protocol. Briefly, the HUVECs were seeded in 6-well plates at a density of $2 \times 10^{5}$ cells/well, and upon reaching $70-80 \%$ confluency, the cells were starved in serum-free medium for $6 \mathrm{~h}$, and then treated with Ang II
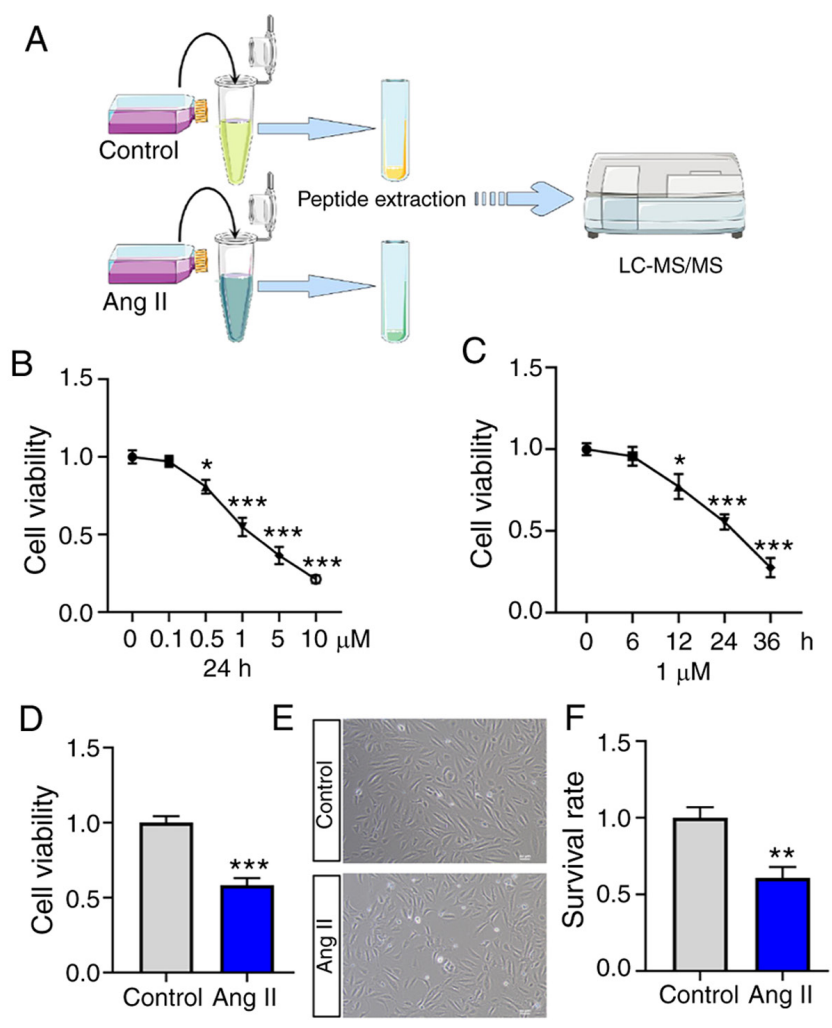

Figure 1. Process of peptidomics research and the construction of a vascular endothelial injury model. (A) Schematic diagram of peptide identification. (B) The changes in cell viabilities were evaluated in HUVECs treated with Ang II at different concentrations $(0.0,0.1,0.5,1.0,5.0$ and $10.0 \mu \mathrm{M})$ for $24 \mathrm{~h}$. (C) The changes in cell viabilities were evaluated in HUVECs treated with Ang II at $1 \mu \mathrm{M}$ concentration for different time $(0,6,12,24$ and $36 \mathrm{~h})$. (D) A stable vascular endothelial injury model was constructed in vitro using $1 \mu \mathrm{M}$ Ang II treatment for $24 \mathrm{~h}$. (E) The cell survival rate was markedly decreased in the Ang II treatment group. (F) Quantitative assessment of cell survival rate. The data are presented as the means $\pm \mathrm{SD} .{ }^{*} \mathrm{P}<0.05^{* *} \mathrm{P}<0.01$ and ${ }^{* * * *} \mathrm{P}<0.001$ vs. control group. HUVECs, human umbilical vein endothelial cells; LC, liquid chromatography; MS, mass spectrometry.

$(1 \mu \mathrm{M})$ for $24 \mathrm{~h}$ at $37^{\circ} \mathrm{C}$; the peptides $(20 \mu \mathrm{M})$ were administered $2 \mathrm{~h}$ before Ang II treatment under the same conditions. Then, the cells were incubated with serum-free medium including $0.1 \% \mathrm{DCFH}-\mathrm{DA}$ away from light at $37^{\circ} \mathrm{C}$ for $20 \mathrm{~min}$, and then washed with serum-free medium three times. Images were captured using a fluorescence microscope (BX61; Olympus Corporation) and the intensity of ROS fluorescence was quantified using ImageJ software 1.26 (National Institutes of Health). The HUVECs were seeded in 6-well plate at a density of $2 \times 10^{5}$ cells/well. After the drug treatment, the cells were digested with trypsin, lysed in RIPA buffer (cat. no. P0013C; Beyotime Institute of Biotechnology) and centrifuged $\left(12,000 \mathrm{x} \mathrm{g}, 4^{\circ} \mathrm{C}\right)$ for $10 \mathrm{~min}$. Commercial assay kits were used to detect the activity of SOD (cat. no. A001-3-1; Nanjing Jiancheng Bioengineering Institute) and the content of MDA (cat. no. A003-3-1; Nanjing Jiancheng Bioengineering Institute) in cells, according to the manufacturer's instructions.

Mitochondrial membrane potential (MMP) detection. The MMP was detected using the JC-1 MMP assay kit (cat. no. C2006; Beyotime Institute of Biotechnology) according to the manufacturer's instructions. The HUVECs were seeded in a 6 -well plate at a density of $2 \times 10^{5}$ cells/well, 

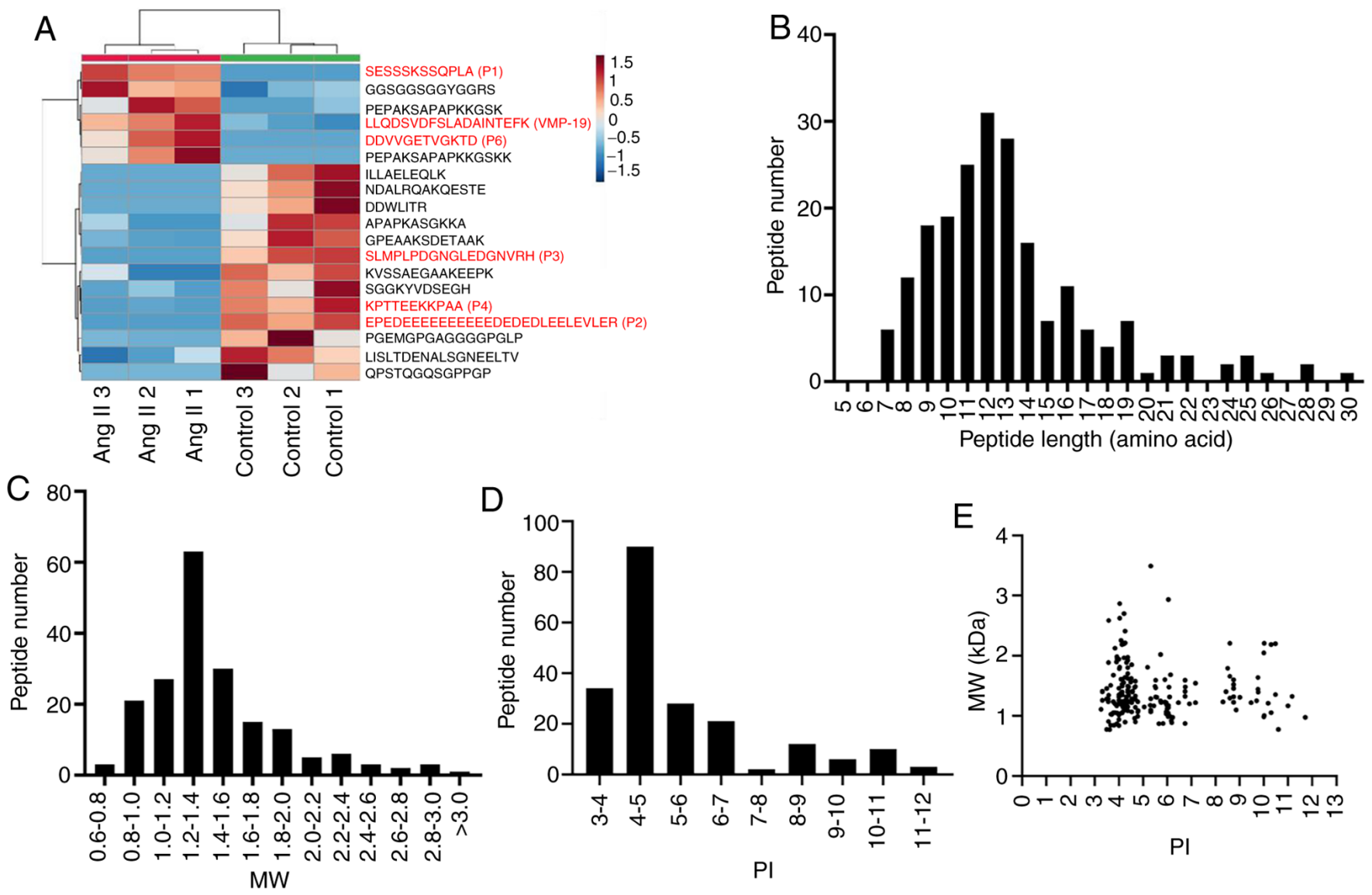

Figure 2. Identification of peptide expression profiles. (A) The heat map shows the significant differences in the peptide profiles of cell supernatants treated with Ang II. (B) The distribution of peptide length. (C) The distribution of the MW of peptides. (D) The PI distribution of the peptides. (E) Correlation between the distribution of MW and PI of peptides. MW, molecular weight; PI, isoelectric point.

and treated with drugs once they reached $70-80 \%$ confluency. After $24 \mathrm{~h}$, the cells were washed with PBS and incubated with JC solution for $10 \mathrm{~min}$ at $37^{\circ} \mathrm{C}$. Cells were then washed with serum-free medium three times and analyzed on a Laser Scanning Confocal Microscope.

Western blotting. The proteins of the HUVECs were extracted using lysate buffer including RIPA buffer (cat. no. P0013K; Beyotime Institute of Biotechnology) and 1\% PMSF (cat. no. ST506; Beyotime Institute of Biotechnology), and the protein concentration was determined using a BCA detection kit (cat. no. 23229; Thermo Fisher Scientific). Following protein extraction, $1 \mathrm{X}$ SDS loading buffer was added to the samples, followed by denaturation by boiling at $95^{\circ} \mathrm{C}$ for $5 \mathrm{~min}$. After cooling for $5 \mathrm{~min}$ on ice, the same amount of protein sample $(20 \mathrm{mg})$ was separated via SDS-PAGE (10\% gel), and then separated proteins were transferred onto PVDF membranes (EMD Millipore). The membrane was blocked with 5\% skimmed milk for $2 \mathrm{~h}$ at room temperature and incubated with anti-poly (ADP-ribose) polymerase 1 (PARP; 1:1,000; cat. no. 9542; Cell Signaling Technology, Inc.), anti-cleaved-PARP (1:1,000; cat. no. 9541; Cell Signaling Technology, Inc.), anti-Caspase3 (1:1,000; cat. no. 9662; Cell Signaling Technology, Inc.), anti-cleaved-Caspase3 (1:1,000; cat. no. 9661; Cell Signaling Technology, Inc.) and anti- $\beta$-actin (1:2,000; cat. no. 4970; Cell Signaling Technology, Inc.) overnight at $4{ }^{\circ} \mathrm{C}$.
After that, the membrane was washed three times for $10 \mathrm{~min}$ each time with TBS with $0.1 \%$ Tween-20 (cat. no. ST825; Beyotime Institute of Biotechnology) buffer and incubated with horseradish peroxidase-conjugated secondary antibodies (anti-rabbit/mouse; 1:3,000; cat. nos. 7074 and 7076; Cell Signaling Technology, Inc.) for $1 \mathrm{~h}$ at room temperature. Image Lab 6 software (Bio-Rad Laboratories, Inc.) was utilized for the semi-quantification of protein expression.

Statistical analysis. All data were obtained from $\geq 3$ individual experiments. GraphPad Prism 8 software (GraphPad Software, Inc.) was utilized to analyze the experimental data and produce the statistical graphs. The data are presented as the means \pm standard deviation. An unpaired two-sided Student's t-test or one-way ANOVA with Bonferroni's correction for multiple comparisons were used to analyze statistical differences. $\mathrm{P}<0.05$ was considered to indicate a statistically significant difference.

\section{Results}

Process of peptidomics research and the construction of a vascular endothelial injury model. The present study used HUVECs to construct a vascular endothelial injury model by treating the cells with Ang II and then collecting the cellular supernatant to extract peptides for MS analysis. A 
Table I. Details of 19 differentially expressed peptides (Ang II vs. Control).

\begin{tabular}{|c|c|c|c|c|c|}
\hline Peptide sequence & $\begin{array}{l}\text { UniProt } \\
\text { accession }\end{array}$ & Protein name & P-value & Fold-change & Regulation \\
\hline SESSSKSSQPLA & P17096 & $\begin{array}{l}\text { High mobility group protein } \\
\text { HMG-I (HMGA1_HUMAN) }\end{array}$ & $5.74526 \times 10^{-5}$ & 1960.78 & Up \\
\hline $\begin{array}{l}\text { EPEDEEEEEEEEEEDEDED } \\
\text { LEELEVLER }\end{array}$ & Q9NQC3 & Reticulon-4 (RTN4_HUMAN) & $1.78342 \times 10^{-4}$ & 9.15 & Down \\
\hline SLMPLPDGNGLEDGNVRH & Q9ULZ1 & Apelin (APEL_HUMAN) & $1.74441 \times 10^{-3}$ & 16.62 & Down \\
\hline КРТTEEKКРАA & P36578 & $\begin{array}{l}\text { 60S ribosomal protein L4 } \\
\text { (RL4_HUMAN) }\end{array}$ & $2.99364 \times 10^{-3}$ & 69.51 & Down \\
\hline LLQDSVDFSLADAINTEFK & P08670 & Vimentin (VIME_HUMAN) & $3.68230 \times 10^{-3}$ & 3.82 & Up \\
\hline DDVVGETVGKTD & P27816 & $\begin{array}{l}\text { Microtubule-associated protein } 4 \\
\text { (MAP4_HUMAN) }\end{array}$ & $7.81321 \times 10^{-3}$ & 53.48 & Up \\
\hline GPEAAKSDETAAK & P04792 & $\begin{array}{l}\text { Heat shock protein } \beta-1 \\
\text { (HSPB1_HUMAN) }\end{array}$ & $1.01935 \times 10^{-2}$ & 3.91 & Down \\
\hline NDALRQAKQESTE & P08670 & Vimentin (VIME_HUMAN) & $1.22044 \times 10^{-2}$ & 26.18 & Down \\
\hline GGSGGSGGYGGRS & P22626 & $\begin{array}{l}\text { Heterogeneous nuclear } \\
\text { ribonucleoproteins A2 } \\
\text { (ROA2_HUMAN) }\end{array}$ & $1.36942 \times 10^{-2}$ & 4.34 & Up \\
\hline ILLAELEQLK & P08670 & Vimentin (VIME_HUMAN) & $1.37267 \times 10^{-2}$ & 172.22 & Down \\
\hline PEPAKSAPAPKKGSKK & O60814 & $\begin{array}{l}\text { Histone H2B type } 1-\mathrm{K} \\
\text { (H2B1K_HUMAN) }\end{array}$ & $1.56135 \times 10^{-2}$ & 42.81 & Up \\
\hline KVSSAEGAAKEEPK & P05114 & $\begin{array}{l}\text { Non-histone chromosomal } \\
\text { protein HMG-14 } \\
\text { (HMGN1_HUMAN) }\end{array}$ & $1.74828 \times 10^{-2}$ & 5.38 & Down \\
\hline DDWDLITR & Q9UBU8 & $\begin{array}{l}\text { Mortality factor 4-like protein } 1 \\
\text { (MO4L1_HUMAN) }\end{array}$ & $1.83306 \times 10^{-2}$ & 68.25 & Down \\
\hline LISLTDENALSGNEELTVK & P14625 & Endoplasmin (ENPL_HUMAN) & $1.95552 \times 10^{-2}$ & 4.30 & Down \\
\hline PEPAKSAPAPKKGSK & O60814 & $\begin{array}{l}\text { Histone H2B type } 1-\mathrm{K} \\
\text { (H2B1K_HUMAN) }\end{array}$ & $2.49500 \times 10^{-2}$ & 15.44 & Up \\
\hline APAPKASGKKA & P50914 & $\begin{array}{l}\text { 60S ribosomal protein L14 } \\
\text { (RL14_HUMAN) }\end{array}$ & $2.60081 \times 10^{-2}$ & 8.82 & Down \\
\hline SGGKYVDSEGH & Q03135 & Caveolin-1 (CAV1_HUMAN) & $2.80822 \times 10^{-2}$ & 7.99 & Down \\
\hline QPSTQGQSGPPGP & 1 & / & $4.29166 \times 10^{-2}$ & 14.72 & Down \\
\hline PGEMGPGAGGGGPGLP & Q96JN8 & $\begin{array}{l}\text { Neuralized-like protein } 4 \\
\text { (NEURL4_HUMAN) }\end{array}$ & $4.81683 \times 10^{-2}$ & 40.51 & Down \\
\hline
\end{tabular}

schematic diagram is presented in Fig. 1A. To construct a stable vascular endothelial injury model, the present study evaluated the changes of cell viabilities in HUVECs treated with Ang II at different concentrations $(0.0,0.1,0.5,1.0$, 5.0 and $10.0 \mu \mathrm{M})$ for different times $(0,6,12,24$ and $36 \mathrm{~h})$ in vitro (Fig. $1 \mathrm{~B}$ and $\mathrm{C}$ ). As demonstrated in Fig. 1D, a stable vascular endothelial injury model was constructed in vitro using $1 \mu \mathrm{M}$ Ang II treatment for $24 \mathrm{~h}$. In addition, the cell survival rate was significantly decreased in the Ang II treatment group (Fig. 1E and F).

Identification of peptide expression profiles. The MS results revealed that a total of 211 peptides were identified from the cell supernatant, of which, six were upregulated and 13 were downregulated when compared with the control group ( $\mathrm{P}<0.05$ and fold-change $\geq 2$ ) (Table I). The heat map shows the significant differences in the peptide profiles of cell supernatants treated with Ang II (Fig. 2A). Through the UniProt database analysis, it was revealed that 57 peptides had no precursor proteins (Table SI). Subsequently, the present study analyzed the physicochemical properties of the peptides that were identified. It was revealed that the distribution of the 211 peptides making up the length of the peptide was mainly concentrated in 9-14 amino acids (Fig. 2B). The present study also analyzed the MW and PI of peptides, and revealed that the MW of peptides were between 1.2 and $1.4 \mathrm{kDa}$, and the PI values were mainly in the PI 4-5 range (Fig. 2C and D). In addition, the present study investigated the association between the distribution of MW and PI of peptides. As presented in Fig. 2E, the peptides were mainly clustered into three groups: Near PI 4, PI 6 and PI 9.

Bioinformatics analysis. Through the UniProt database analysis, a total of 154 peptides were derived from 62 precursor 
A

Molecular function

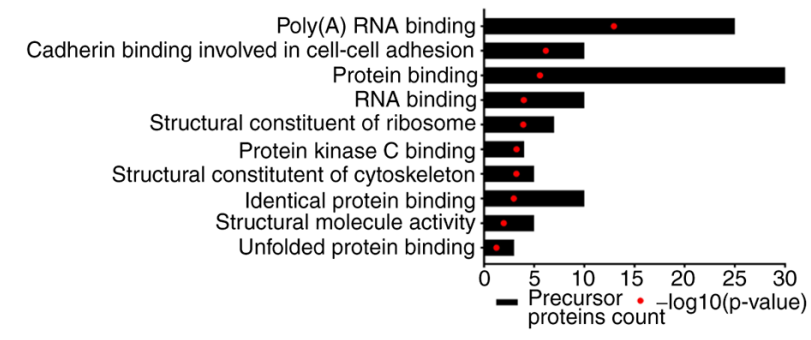

C

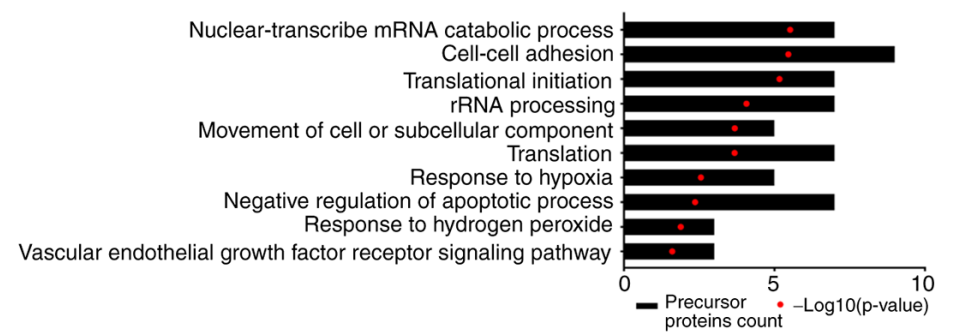

B

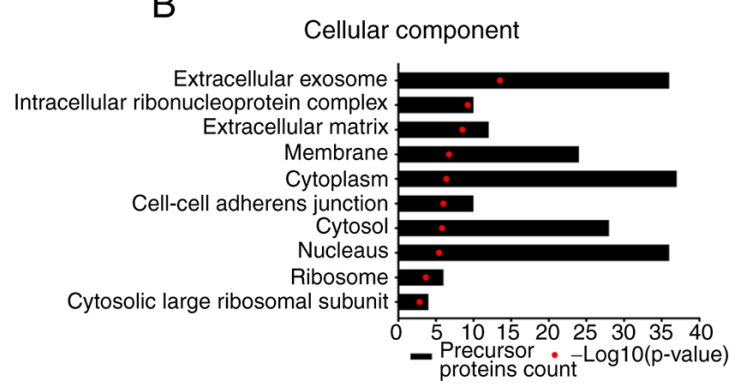

D

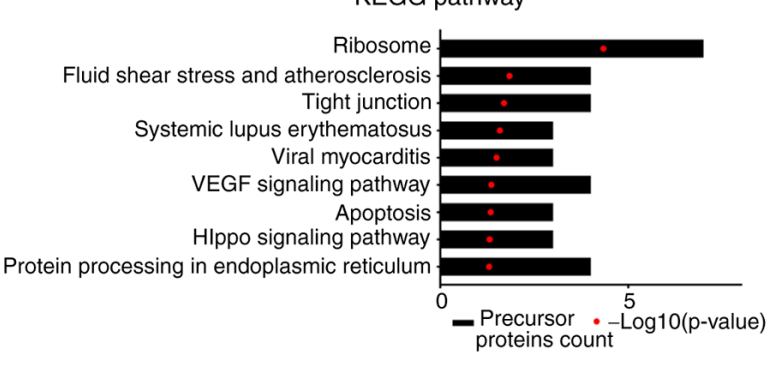

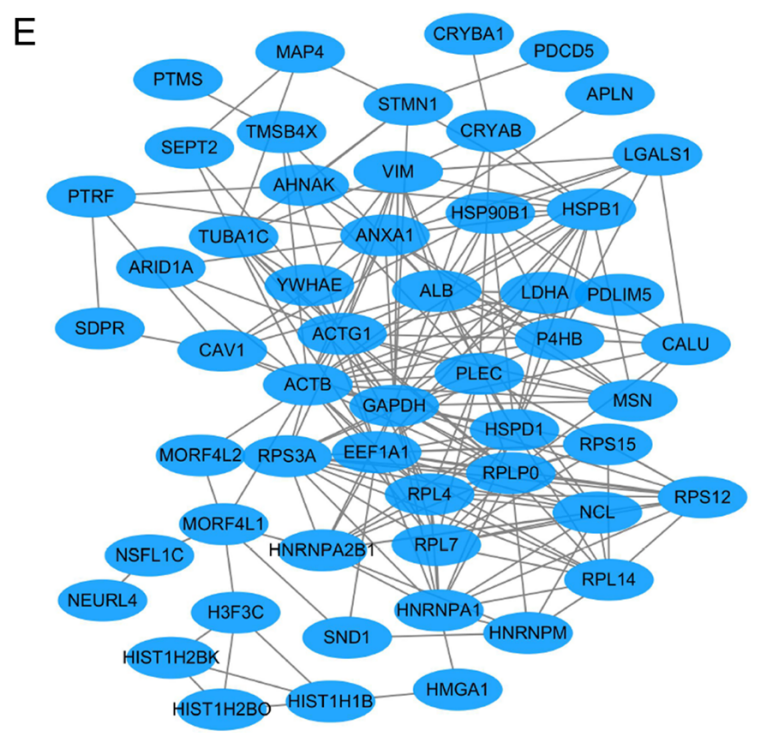

Figure 3. Bioinformatics analysis of peptide precursor proteins. Gene Ontology analysis of (A) 'Molecular function', (B) 'Cellular component' and (C) 'Biological process'. (D) KEGG pathway analysis. (E) A typical Search Tool for the Retrieval of Interacting Genes/Proteins network interaction diagram of these peptide precursor proteins, medium confidence 0.400. KEGG, Kyoto Encyclopedia of Genes and Genomes.

proteins, and 57 peptides were found to have no precursor proteins. To predict the potential function of these identified peptides, the present study performed GO and KEGG pathway analyses on their precursor proteins. The GO analysis results revealed that the 10 'Molecular function' categories most enriched with these peptides were 'Poly(A) RNA binding', 'Cadherin binding involved in cell-cell adhesion', 'Protein binding', 'RNA binding', 'Structural constituent of ribosome', 'Protein kinase C binding', 'Structural constituent of cytoskeleton', 'Identical protein binding', 'Structural molecule activity' and 'Unfolded protein binding' (Fig. 3A). The top 10 most enriched subcellular localization categories were 'Extracellular exosome', 'Intracellular ribonucleoprotein complex', 'Extracellular matrix', 'Membrane', 'Cytoplasm', 'Cell-cell adherens junction', 'Cytosol', 'Nucleus', 'Ribosome' and 'Cytosolic large ribosomal subunit' (Fig. 3B). The 'Biological function' categories most enriched with these precursors were mainly associated with 'Nuclear-transcribed mRNA catabolic process', 'Cell-cell adhesion', 'Translational initiation', 'rRNA processing', 'Movement of cell or subcellular component', 'Translation', 'Response to hypoxia', 'Negative regulation of apoptotic process', 'Response to hydrogen peroxide' and 'Vascular endothelial growth factor receptor signaling pathway' (Fig. 3C). The KEGG pathway analysis showed that the precursor proteins were mainly associated with 'Ribosome', 'Fluid shear stress and atherosclerosis', 'Tight junction', 'Systemic lupus erythematosus', 'Viral myocarditis', 'VEGF signaling pathway', 'Apoptosis', 'Hippo signaling pathway' and 'Protein processing in endoplasmic reticulum' (Fig. 3D). In addition, the present study also analyzed the interaction network of these peptide precursor proteins using the STRING website. A typical STRING network interaction diagram is presented in Fig. 3E. 

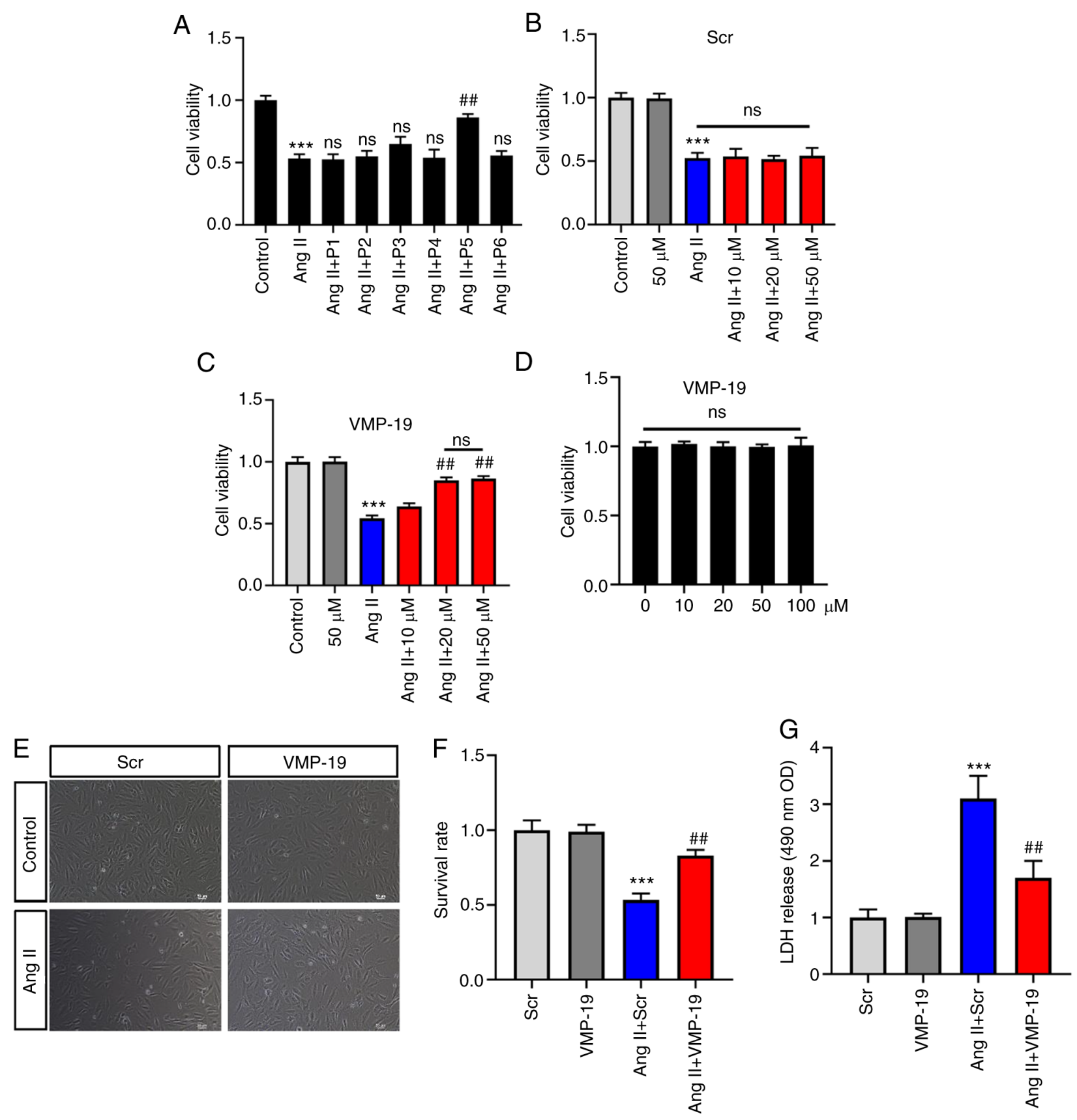

Figure 4. Preliminary functional exploration of peptides. (A) Preliminary screening of functional peptides by evaluating cell viability. (B) The Scr had no significant effects on cell viability with Ang II treatment. (C) VMP-19 could significantly increase the cell viability induced by Ang II in a concentration-dependent manner, and the optimum effect was at $20 \mu \mathrm{M}$. (D) VMP-19 peptide had no toxic effect at high concentrations in human umbilical vein endothelial cells. (E) Cell survival was evaluated. Magnification, x100. (F) Quantitative assessment of cell survival rate. (G) Effects of $20 \mu \mathrm{M}$ VMP-19 on LDH release caused by Ang II treatment. $\mathrm{n}=3$ /group. The data are presented as the means $\pm \mathrm{SD}$. ${ }^{* * *} \mathrm{P}<0.001$ vs. the control group; ${ }^{\# /} \mathrm{P}<0.01 \mathrm{vs}$. Ang II or Ang II+Scr group. Scr, scrambled peptide; ns, not statistically significant; LDH, lactate dehydrogenase.

Preliminary functional investigation of peptides. To identify the peptides with vascular endothelial protection function, the present study selected the top six peptides from 19 differential peptides according to the P-value. The sequences of the six peptides are presented in Table SII. The results revealed that the peptide with the LLQDSVDFSLADAINTEFK sequence could significantly increase the cell viability induced by Ang II (Fig. 4A). According to the name of its precursor protein and the number of amino acids, this peptide was named VMP-19 (a peptide derived from Vimentin, 19 amino acids) in the present study. In addition, the present study assessed the effects of different concentrations of VMP-19 and its Scr on cell viability. As a control peptide, there was no significant difference in the cell viability between the Scr and Ang II treatment (Fig. 4B). However, VMP-19 could significantly increase the cell viability induced by Ang II in a concentration-dependent manner, and the effect was most obvious at $20 \mu \mathrm{M}$ (Fig. 4C). The present study also evaluated the effects of the VMP-19 peptide itself on the cell viability of HUVECs. The results revealed that VMP-19 had no toxic effect at high concentrations in HUVECs (Fig. 4D). Cell survival was also evaluated, and it was revealed that $20 \mu \mathrm{M}$ 

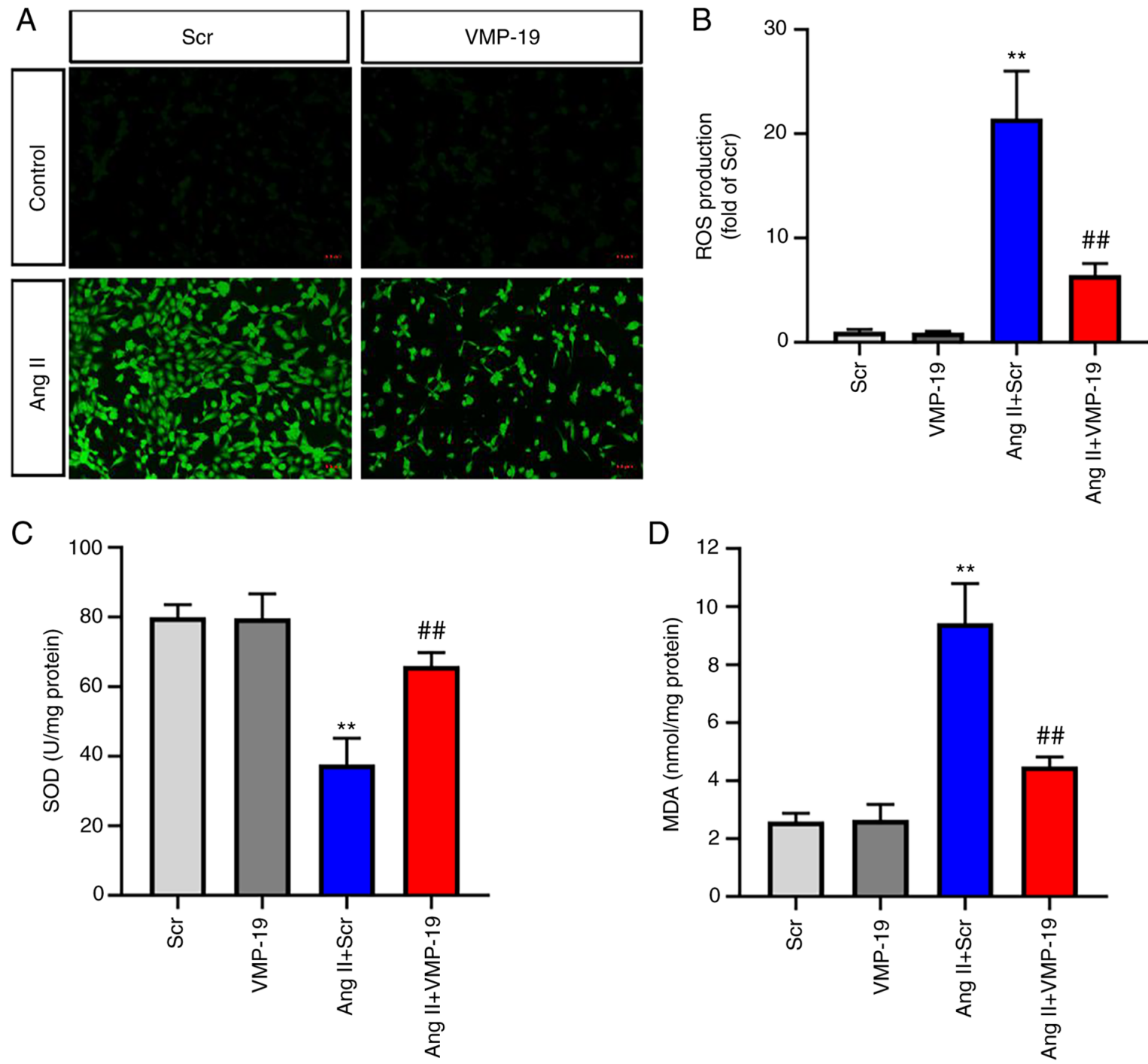

Figure 5. VMP-19 peptide attenuates oxidative stress induced by Ang II. (A) Representative images of the ROS staining. Intracellular ROS was detected by DCFH-DA. Green, ROS. Magnification, x100. (B) Quantification analysis of ROS. Detection of (C) cellular SOD activity and (D) cellular MDA content. $\mathrm{n}=3$ /group. The data are presented as the means $\pm \mathrm{SD}$. ${ }^{* *} \mathrm{P}<0.01$ vs. the control group; ${ }^{\# \#} \mathrm{P}<0.01$ vs. Ang II+Scr group. ROS, reactive oxygen species; SOD, superoxide dismutase; MDA, malondialdehyde; Scr, scrambled peptide.

VMP-19 significantly increased the survival rate of HUVECs with treatment of Ang II (Fig. 4E and F). LDH is an important indicator of cell damage. In the present study, $20 \mu \mathrm{M}$ VMP-19 could significantly decrease the release of LDH caused by Ang II treatment (Fig. 4G). The aforementioned results indicated that VMP-19 exerts protection in Ang II-induced HUVEC damage in a dose-dependent manner, and the effect was most obvious at $20 \mu \mathrm{M}$.

VMP-19 peptide attenuates oxidative stress induced by Ang II. Considering that the excessive production of ROS is the main biological event of Ang II-induced HUVECs injury, ROS were examined via DCFH-DA staining. As presented in Fig. 5A and B, $20 \mu \mathrm{M}$ VMP-19 significantly decreased the production of ROS after Ang II treatment in HUVECs. In addition, the present study also measured the activities of major antioxidant enzymes: SOD and the content of MDA. A dose of $20 \mu \mathrm{M}$ VMP-19 peptide significantly increased the activities of SOD and decreased the content of MDA after Ang II treatment in HUVECs (Fig. 5C and D).
VMP-19 peptide attenuates apoptosis induced by Ang II. Apoptosis of VECs is a vital event in the development of endothelial dysfunction. The decrease of MMP is a sign of early apoptosis. In the present study, VMP-19 significantly improved the decrease of MMP by Ang II in HUVECs, as evidenced by the ratio of aggregated JC-1 monomer (Fig. 6A and B). In order to further investigate the effect of VMP-19 on Ang II-induced HUVEC apoptosis injury, the proteins associated with apoptosis were evaluated. The activation of PARP and caspase 3 was inhibited following VMP-19 peptide treatment in Ang II-induced cell damage (Fig. 6C-E). Hence, the present study confirmed that VMP-19 peptide could protect HUVECs from apoptosis induced by Ang II.

\section{Discussion}

It is already well-established that VECs are important metabolic and endocrine organs that play an important role in regulating vascular function, and vascular endothelial injury 
A
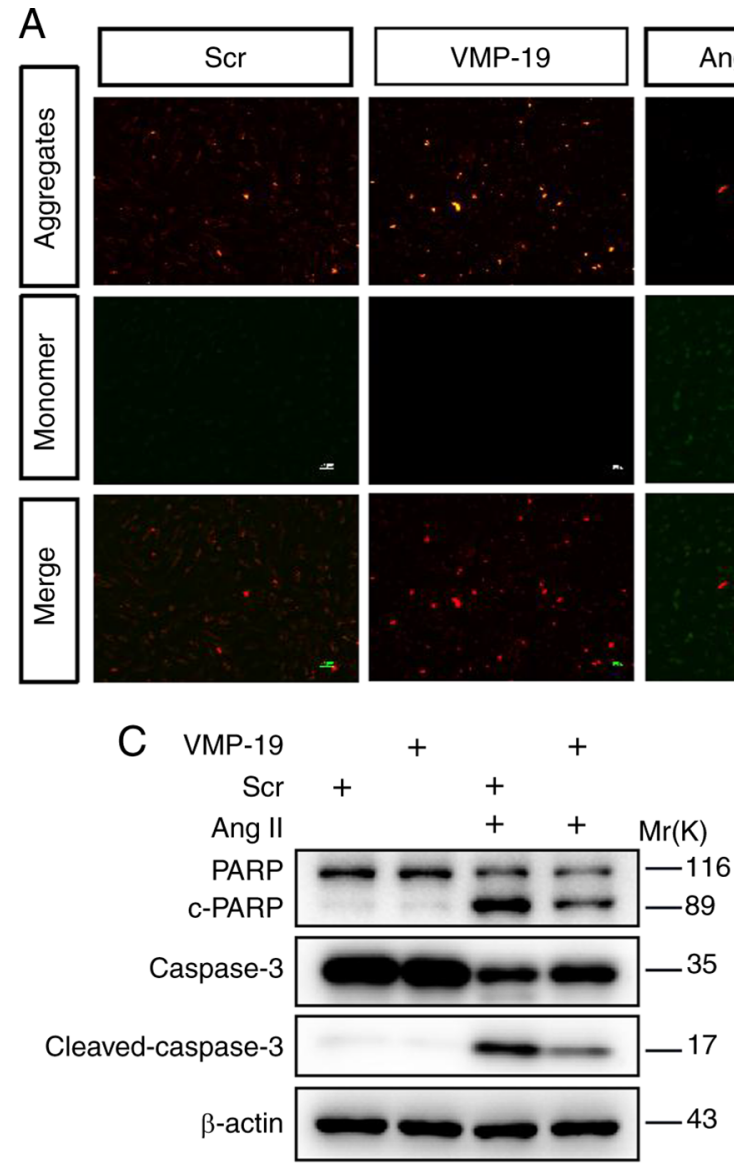
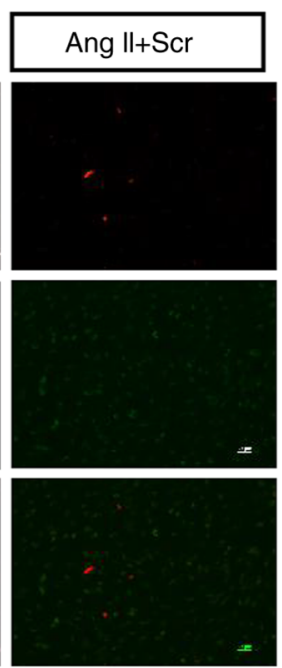

D

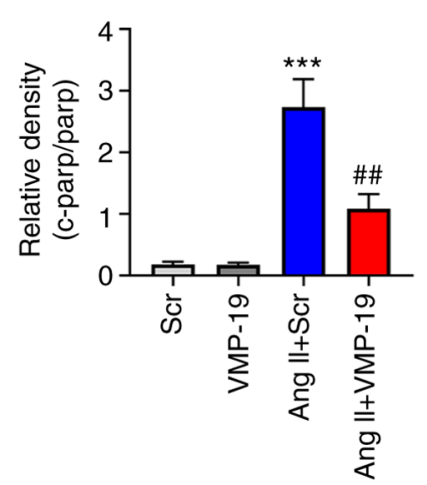

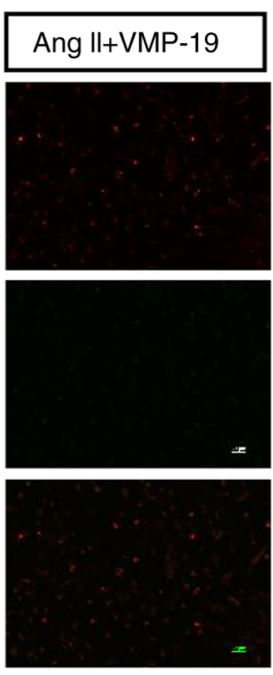

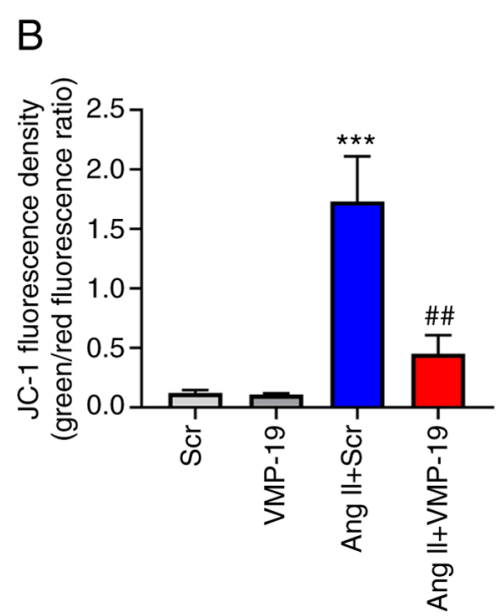

$E$ ल

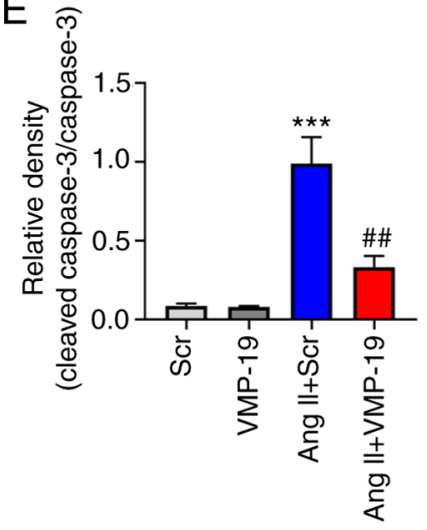

Figure 6. VMP-19 peptide attenuates apoptosis induced by Ang II. (A) Representative images of mitochondrial membrane potential. Magnification, x100. (B) Quantification analysis of JC-1 fluorescence intensity. (C) The proteins associated with apoptosis (PARP and caspase3) were measured via western blotting. Semi-quantitative analysis of (D) PARP and (E) caspase3. $n=3$ /group. The data are presented as the means \pm SD. ${ }^{* * *} \mathrm{P}<0.001$ vs. control group; ${ }^{\#} \mathrm{P}<0.01$ vs. Ang II+Scr group. Scr, scrambled peptide; PARP, poly (ADP-ribose) polymerase 1; c-, cleaved.

is closely associated with the development and progression of various cardiovascular diseases, particularly hypertension (25). Therefore, focusing on the alleviation of vascular endothelial injury will help to prevent and treat hypertension. However, at present, there is no effective prevention and treatment strategy for vascular endothelial injury. In the present study, proteomics methods were used to identify the peptides secreted by HUVECs under Ang II stimulation. A total of 211 secreted peptides were identified, of which 19 were significantly differentially expressed. Through further experiments, a VMP-19 peptide was identified to alleviate the apoptosis and oxidative stress injury in HUVECs. The results indicated that VMP-19 peptide may be a candidate molecule for the treatment of vascular endothelial injury.

As HUVECs exhibit a similar human physiological state and have similar biological characteristics to VECs, no species difference, rich sources and ethical advantages, they are widely used as cell models to simulate hypertension injury (26). A large number of studies have revealed that Ang II, as the main effector peptide of the renin-angiotensin-aldosterone system, is closely associated with the occurrence and development of hypertension $(27,28)$. Therefore, stimulation of HUVECs with Ang II is a reliable model to simulate vascular endothelial injury in vitro. In previous studies, the concentration and dose time of Ang II in an in vitro model were different $(29,30)$. As presented in Fig. 1B-D, the present study used HUVECs to construct a stable vascular endothelial model with $1 \mu \mathrm{M}$ Ang II treatment for $24 \mathrm{~h}$ in vitro. Similarly, the same in vitro model method was used to verify the protective effect of E3 ubiquitin-protein ligase NEDD4 on Ang II-induced vascular endothelial cell injury by regulating exportin-1-mediated nuclear export (31).

The present study identified a total of 211 peptides from the cell supernatant. The length of these peptides was mainly concentrated in 9-14 amino acids, and the MW was distributed between 1.2 and $1.4 \mathrm{kDa}$, and $<3.0 \mathrm{kDa}$, which suggested that the peptides identified in the present study were valid. Among the identified peptides, a number of them were derived from the same precursor protein. It is already well-known that the majority of peptides are produced by protein cleavage into fragments, and proteases play a vital role in the protein cleavage process by recognizing the cleavage site specifically (32). Notably, the present study revealed that the precursor proteins of 57 of the identified peptides were not found in the UniProt database. A previous study shown that some non-coding RNAs with short open reading frames 
have been verified to possess coding ability and can exert their functions via encoding peptides (33). For instance, a peptide encoded by a putative lncRNAHOXB-AS3 suppresses colon cancer growth through competitively binding to the arginine residues in the RGG motif of heterogenous nuclear ribonucleoprotein A1 (34). In addition, the LINC00961-encoded SPAR peptide could regulate $\mathrm{mTORC} 1$ and muscle regeneration (35). Therefore, the present study speculated that these peptides may also be encoded by some non-coding RNAs, which will be clarified in future research.

The underlying mechanism of vascular endothelial injury mainly involves endothelial cell apoptosis, inflammation and oxidative stress injury $(36,37)$. The present study focused on the role of peptides in VEC apoptosis and oxidative stress injury, and successfully identified a peptide named VMP-19 with the protective functions of VECs. The results demonstrated that the VMP-19 peptide could alleviate the apoptosis and oxidative stress injury of VECs induced by Ang II, as indicated by a decreased level of the proteins associated with apoptosis and production of ROS. Some precursor proteins and their derived peptides may play the same role in certain diseases. The VMP-19 peptide is derived from the Vimentin protein, located at amino acid 79-97. A previous study revealed that Vimentin not only maintains cell integrity, but also regulates apoptosis, inflammation and immune response (38). Kueper et al (39) found that Vimentin cleavage is a common phenomenon in the process of apoptosis. Combined with the findings of the present study, we speculated that the Vimentin protein may cleave into a shorter peptide to protect VECs from apoptosis and oxidative stress injury.

Although a novel peptide was identified in the present study, which was named VMP-19, which could protect HUVECs from apoptosis and oxidative stress injury, there were still some limitations to the study. For example, whether different modification methods could affect the function of VMP-19 requires further verification. In addition, the specific mechanism underlying the VMP-19 peptide in the protection of VECs remains to be verified. Therefore, in future work, the effect of the VMP-19 peptide under different modification conditions will be investigated in order to clarify the specific mechanism of its vascular endothelial protection.

In summary, to the best of our knowledge, the present study was the first to use peptidomics to analyze the peptide spectrum of supernatants secreted by HUVECs and identified that a novel peptide, VMP-19, could protect HUVECs from apoptosis and oxidative stress injury. The results of the present study could provide novel insights into the treatment of hypertension.

\section{Acknowledgements}

Not applicable.

\section{Funding}

The present study was funded by the Health Commission of Changning District, Shanghai, China (grant no. YXMZK009) and the National Natural Science Foundation of China (grant no. 81873540$)$.

\section{Availability of data and materials}

The datasets used and/or analyzed during the current study are available from the corresponding author on reasonable request.

\section{Authors' contributions}

ZX, JD and LZ performed the experiments and wrote the manuscript. XF and JZ performed the bioinformatics analysis. $\mathrm{XS}$ and HL performed some of the in vitro experiments. XL and LQ contributed to the study design and concept, and supervised the project. ZX and JD confirm the authenticity of all the raw data. All authors read and approved the final manuscript.

\section{Ethics approval and consent to participate}

Not applicable.

\section{Patient consent for publication}

Not applicable.

\section{Competing interests}

The authors declare that they have no competing interests.

\section{References}

1. Humbert M, Montani D, Perros F, Dorfmuller P, Adnot S and Eddahibi S: Endothelial cell dysfunction and cross talk between endothelium and smooth muscle cells in pulmonary arterial hypertension. Vascul Pharmacol 49: 113-118, 2008.

2. Konukoglu D and Uzun H: Endothelial dysfunction and hypertension. Adv Exp Med Biol 956: 511-540, 2017.

3. Cahill PA and Redmond EM: Vascular endothelium-Gatekeeper of vessel health. Atherosclerosis 248: 97-109, 2016.

4. Martelli A, Testai L, Anzini M, Cappelli A, Di Capua A, Biava M, Poce G, Consalvi S, Giordani A, Caselli G, et al: The novel anti-inflammatory agent VA694, endowed with both NO-releasing and COX2-selective inhibiting properties, exhibits NO-mediated positive effects on blood pressure, coronary flow and endothelium in an experimental model of hypertension and endothelial dysfunction. Pharmacol Res 78: 1-9, 2013.

5. Lankhorst S, Kappers MH, van Esch JH, Danser AH and van den Meiracker AH: Hypertension during vascular endothelial growth factor inhibition: Focus on nitric oxide, endothelin-1, and oxidative stress. Antioxid Redox Signal 20: 135-145, 2014

6. Zhang PW, Yu CL, Wang YZ, Luo SF, Sun LS and Li RS: Influence of 3,4',5-trihydroxystibene-3-beta-mono-D-glucoside on vascular endothelial epoprostenol and platelet aggregation. Zhongguo Yao Li Xue Bao 16: 265-268, 1995.

7. Gizard F and Bruemmer D: Transcriptional control of vascular smooth muscle cell proliferation by peroxisome proliferator-activated receptor-gamma: Therapeutic implications for cardiovascular diseases. PPAR Res 2008: 429123, 2008.

8. Carney EF: Hypertension: New non-RAS peptide modulates the vasoregulatory effects of angiotensin II. Nat Rev Nephrol 11: 317, 2015.

9. Ceriello A, Novials A, Ortega E, Canivell S, La Sala L, Pujadas G, Esposito K, Giugliano D and Genovese S: Glucagon-like peptide 1 reduces endothelial dysfunction, inflammation, and oxidative stress induced by both hyperglycemia and hypoglycemia in type 1 diabetes. Diabetes Care 36: 2346-2350, 2013.

10. Chaturvedi LS and Basson MD: Glucagonlike peptide 2 analogue teduglutide: Stimulation of proliferation but reduction of differentiation in human Caco-2 intestinal epithelial cells. JAMA Surg 148: 1037-1042, 2013. 
11. Dang LT, Feric NT, Laschinger C, Chang WY, Zhang B, Wood GA, Stanford WL and Radisic M: Inhibition of apoptosis in human induced pluripotent stem cells during expansion in a defined culture using angiopoietin-1 derived peptide QHREDGS. Biomaterials 35: 7786-7799, 2014.

12. Fosgerau K and Hoffmann T: Peptide therapeutics: Current status and future directions. Drug Discov Today 20: 122-128, 2015.

13. Kaspar AA and Reichert JM: Future directions for peptide therapeutics development. Drug Discov Today 18: 807-817, 2013.

14. Anand IS, Fisher LD, Chiang YT, Latini R, Masson S, Maggioni AP, Glazer RD, Tognoni G and Cohn JN; Val-HeFT Investigators: Changes in brain natriuretic peptide and norepinephrine over time and mortality and morbidity in the Valsartan heart failure trial (Val-HeFT). Circulation 107: 1278-1283, 2003.

15. Armstrong PW and Rouleau JL: A canadian context for the acute study of clinical effectiveness of nesiritide and decompensated heart failure (ASCEND-HF) trial. Can J Cardiol 24 (Suppl B): 30B-32B, 2008.

16. Cerrato BD, Carretero OA, Janic B, Grecco HE and Gironacci MM: Heteromerization between the bradykinin B2 receptor and the angiotensin-(1-7) mas receptor: Functional consequences. Hypertension 68: 1039-1048, 2016.

17. Koid SS, Ziogas J and Campbell DJ: Aliskiren reduces myocardial ischemia-reperfusion injury by a bradykinin B2 receptor- and angiotensin AT2 receptor-mediated mechanism. Hypertension 63: 768-773, 2014.

18. Mughal A and O'Rourke ST: Vascular effects of apelin: Mechanisms and therapeutic potential. Pharmacol Ther 190: 139-147, 2018

19. Dallas DC, Guerrero A, Parker EA, Robinson RC, Gan J, German JB, Barile D and Lebrilla CB: Current peptidomics: Applications, purification, identification, quantification, and functional analysis. Proteomics 15: 1026-1038, 2015.

20. Slavoff SA, Mitchell AJ, Schwaid AG, Cabili MN, Ma J, Levin JZ, Karger AD, Budnik BA, Rinn JL and Saghatelian A: Peptidomic discovery of short open reading frame-encoded peptides in human cells. Nat Chem Biol 9: 59-64, 2013.

21. Rubakhin SS, Churchill JD, Greenough WT and Sweedler JV: Profiling signaling peptides in single mammalian cells using mass spectrometry. Anal Chem 78: 7267-7272, 2006.

22. Ashburner M, Ball CA, Black JA, Botstein D, Butler H, Cherry JM, Davis AP, Dolinski K, Dwight SS, Eppig JT, et al: Gene ontology: Tool for the unification of biology: The Gene ontology Consortium. Nat Genet 25: 25-29, 2000.

23. Gene Ontology Consortium: The Gene Ontology resource: Enriching a GOld mine. Nucleic Acids Res 49: D325-D334, 2021.

24. Kanehisa M: Toward pathway engineering: A new database of genetic and molecular pathways. Sci Technol Japan 59: 34-38, 1996.

25. Liu S, Yi F, Cheng W, Qu X and Wang C: Molecular mechanisms in vascular injury induced by hypertension: Expression and role of microRNA-34a. Exp Ther Med 14: 5497-5502, 2017.

26. Reichlin T, Wild A, Durrenberger M, Daniels AU, Aebi U, Hunziker PR and Stolz M: Investigating native coronary artery endothelium in situ and in cell culture by scanning force microscopy. J Struct Biol 152: 52-63, 2005.

27. Biancardi VC, Bomfim GF, Reis WL, Al-Gassimi S and Nunes KP: The interplay between Angiotensin II, TLR4 and hypertension. Pharmacol Res 120: 88-96, 2017.
28. Cha SA, Park BM and Kim SH: Angiotensin-(1-9) ameliorates pulmonary arterial hypertension via angiotensin type II receptor. Korean J Physiol Pharmacol 22: 447-456, 2018.

29. Yang Y, Tian T, Wang Y,Li Z, Xing K and Tian G: SIRT6 protects vascular endothelial cells from angiotensin II-induced apoptosis and oxidative stress by promoting the activation of Nrf2/ARE signaling. Eur J Pharmacol 859: 172516, 2019.

30. Song J, Huang S, Wang K, Li W, Pao L, Chen F and Zhao X: Long Non-coding RNA MEG3 attenuates the angiotensin II-induced injury of human umbilical vein endothelial cells by interacting with p53. Front Genet 10: 78, 2019.

31. Xu J, Sheng Z, Li F, Wang S, Yuan Y, Wang M and Yu Z: NEDD4 protects vascular endothelial cells against Angiotensin II-induced cell death via enhancement of XPO1-mediated nuclear export. Exp Cell Res 383: 111505, 2019.

32. Li F, Wang Y, Li C, Marquez-Lago TT, Leier A, Rawlings ND, Haffari G, Revote J, Akutsu T, Chou KC, et al: Twenty years of bioinformatics research for protease-specific substrate and cleavage site prediction: A comprehensive revisit and benchmarking of existing methods. Brief Bioinform 20: 2150-2166, 2019.

33. Wu P, Mo Y, Peng M, Tang T, Zhong Y, Deng X, Xiong F, Guo C, Wu X, Li Y, et al: Emerging role of tumor-related functional peptides encoded by lncRNA and circRNA. Mol Cancer 19: 22, 2020.

34. Huang JZ, Chen M, Chen D, Gao XC, Zhu S, Huang H, Hu M, Zhu $\mathrm{H}$ and Yan GR: A peptide encoded by a putative lncRNA HOXB-AS3 suppresses colon cancer growth. Mol Cell 68: 171-184, 2017.

35. Matsumoto A, Pasut A, Matsumoto M, Yamashita R, Fung J, Monteleone E, Saghatelian A, Nakayama KI, Clohessy JG and Pandolfi PP: mTORC1 and muscle regeneration are regulated by the LINC00961-encoded SPAR polypeptide. Nature 541: 228-232, 2017.

36. Ellinsworth DC: Arsenic, reactive oxygen, and endothelial dysfunction. J Pharmacol Exp Ther 353: 458-464, 2015.

37. Wang Q, Zhang M, Ding Y, Wang Q, Zhang W, Song P and Zou MH: Activation of NAD(P)H oxidase by tryptophan-derived 3-hydroxykynurenine accelerates endothelial apoptosis and dysfunction in vivo. Circ Res 114: 480-492, 2014.

38. Yang L, Tang L, Dai F, Meng G, Yin R, Xu X and Yao W: Raf-1/CK 2 and RhoA/ROCK signaling promote TNF- $\alpha$-mediated endothelial apoptosis via regulating vimentin cytoskeleton. Toxicology 389: 74-84, 2017.

39. Kueper T, Grune T, Prahl S, Lenz H, Welge V, Biernoth T, Vogt Y, Muhr GM, Gaemlich A, Jung T, et al: Vimentin is the specific target in skin glycation. Structural prerequisites, functional consequences, and role in skin aging. J Biol Chem 282: 23427-23436, 2007.

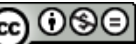

This work is licensed under a Creative Commons

Attribution-NonCommercial-NoDerivatives 4.0 International (CC BY-NC-ND 4.0) License. 